\title{
Comparison of Salmonella enterica serovar Heidelberg Susceptibility Testing Results
}

\author{
Rajesh Nayak, PhD; Veronica Call, BS; Pravin Kaldhone, MS; Cynthia Tyler, MS; \\ Gwendolyn Anderson, BS; Sarah Phillips, PhD; Khalil Kerdahi, BS; and Steven L. Foley, PhD
}

\begin{abstract}
Objective: Disk diffusion and broth dilution assays are conventionally used for antimicrobial susceptibility testing (AST) of bacteria. The goal of this study was to determine the correlation of results from different AST methods for the Salmonella enterica serovar Heidelberg.
\end{abstract}

Design: S. enterica serovar Heidelberg $(n=105)$ strains were tested using 4 different AST methods: agar disk diffusion, broth microdilution using Sensititre with the NARMS (CMVIAGNF) panel, manual broth microdilution and Vitek with GNS-207 cards.

Methods: AST was performed using standardized methods and Clinical and Laboratory Standards Institute recommended quality control organisms. Eight drugs were common to all testing methods including amikacin, amoxicillin/clavulanic acid, ampicillin, chloramphenicol, ciprofloxacin, gentamicin, tetracycline and trimethoprim/sulfamethoxazole.

Results: No resistance to amikacin and ciprofloxacin was detected. Overall, the agreement of the AST results among all four methods for the drugs tested was: amikacin (100\%), amoxicillin/clavulanic acid (96.1\%), ampicillin (97.1\%), chloramphenicol (96.2\%), ciprofloxacin (100\%), gentamicin $(80.0 \%)$, tetracycline (80.0\%) and trimethoprim/sulfamethoxazole (94.3\%). There was $97.1 \%, 95.5 \%$ and $98.0 \%$ overall agreement between the reference diffusion method and the manual broth microdilution, Sensititre microdilution and Vitek methods, respectively.

Conclusion: The study indicated that AST methods correlated with one another when testing $S$. enterica serovar Heidelberg isolates, with a few exceptions. In general, discrepancies among the methods were due to isolates being interpreted as intermediately susceptible or due to an increased number of resistances detected with Sensititre and a lower number with Vitek.

Keywords: Antimicrobial susceptibility testing; Broth microdilution; Disk diffusion;

Salmonella enterica serotype Heidelberg

Reprint Requests:

Steven L. Foley, PhD

Marshfield Clinic Research Foundation

1000 North Oak Avenue

Marshfield, WI 54449

Tel: $715-389-4012$

Fax: 715-389-3808

E-mail: foley.steven@mcrf.mfldclin.edu
Received: October 2, 2006

Revised: December 28, 2006

Accepted: February 21, 2007

doi: $10.312 \mathrm{I} / \mathrm{cmr} \cdot 2007.725$
Grant Support: Support was provided by Marshfield Clinic Research Foundation. The Arkansas Biomedical Research Infrastructure Network is funded by the National Institutes for Health.

Disclaimer: The use of trade names is for identification purposes only and does not imply endorsement by the US Food and Drug Administration or the US Department of Health and Human Services. Views presented in this manuscript do not necessarily reflect those of the FDA. 
S important animal pathogens which are increasingly becoming human pathogens and a considerable public health concern. Each year there are an estimated 1.4 million cases of Salmonella infections in the United States that result in 17,000 hospitalizations and 585 deaths. ${ }^{1}$ Among the Salmonella species responsible for causing human disease, Heidelberg is the fourth most commonly implicated serotype. ${ }^{2}$ Over the past 10 years, the incidence of $S$. enterica serovar Heidelberg infections in humans has increased by $25 \% .^{3}$ Among the food animals, S. enterica serovar Heidelberg is the most commonly identified serotype among isolates originating from turkeys and chickens analyzed by the National Veterinary Services Laboratory. ${ }^{4}$ In addition, S. enterica serovar Heidelberg strains are found throughout the turkey production and processing environments, and in retail turkey products. ${ }^{5-7}$ Because there has been a significant increase in per capita consumption of poultry products over the past 50 years, and because the majority of salmonellosis cases are associated with consumption of contaminated foods, ${ }^{1,8}$ the contamination of poultry products with $S$. enterica serovar Heidelberg has become a major health concern. ${ }^{9}$ Adding to this concern is the emergence and spread of antimicrobial resistant strains of $S$. enterica serovar Heidelberg 9,10 potentially caused by the use of antimicrobial agents in the animal production environment and is threatening the management of salmonellosis in both veterinary and human clinical practice. It is important, therefore, to compare the results of common susceptibility testing methods to facilitate the sharing of susceptibility data from this potentially emerging pathogen.

Antimicrobial susceptibility testing (AST) is critical to clinical and diagnostic microbiology as it can provide insights into treatment options to combat potentially antimicrobial-resistant infectious diseases. The problems of antimicrobial resistance in foodborne pathogens led the US federal government to develop the National Antimicrobial Resistance Monitoring System (NARMS), a multi-agency collaboration between the Food and Drug Administration (FDA), Centers for Disease Control and Prevention (CDC), and Department of Agriculture (USDA). ${ }^{11}$ Through NARMS, antimicrobial resistance is monitored in enteric bacteria isolated from human infections, food animal slaughter and diagnostic samples, and retail foods. ${ }^{5,9}$ The AST method utilized by the NARMS program is a broth microdilution method, which provides a quantitative measure of susceptibility. ${ }^{5}$ Interpretation of the results of broth microdilution and other AST methods is often guided by criteria set by the Clinical Laboratory Standards Institute (CLSI). ${ }^{12}$

Due to their wide application in clinical diagnostics and public health, a number of methods and instrumentation have been developed to carry out AST. ${ }^{13-15}$ With the diversity of testing methods available, most laboratories lack all the different resources (automated and semi-automated systems) to carry out AST. Hence, it is important to determine whether the interpreted results obtained by the different methods correlate with one another to ensure that the results obtained in multiple laboratories could be compared without the time delay and added expense of sending the isolates to a reference laboratory. Time is of the essence in cases of widespread disease outbreaks or potential bioterrorism events, where large numbers of isolates would be screened to determine if they are resistant to the first-line drug therapy. ${ }^{16-20}$ In the current study, we compared the results of four different AST methods (agar disk diffusion method, manual broth microdilution, semi-automated broth microdilution, and Vitek) in S. enterica serovar Heidelberg isolates.

\section{Material and Methods \\ Organisms}

One hundred and five S. enterica serovar Heidelberg isolates $(n=105)$ were screened in this study. ${ }^{7}$ Isolates were obtained from turkeys and turkey production facilities.

\section{Susceptibility Testing Methods}

Four different typing methods were used in this study to evaluate the accuracy of the method to correctly determine the antibiotic susceptibility of $S$. enterica serovar Heidelberg isolates. Each of the methods screened a panel of antimicrobial agents (approximately 15). Among the agents tested, eight drugs were common in all four methods and were used for the evaluating the accuracy of the different AST methods. Furthermore, as each method has different measurement endpoints, we collectively interpreted the results based on whether a bacterial strain was susceptible, intermediate-susceptible or resistant to the antimicrobial agent. The eight common drugs evaluated were amikacin, amoxicillin/clavulanic acid, ampicillin, chloramphenicol, ciprofloxacin, gentamicin, tetracycline and trimethoprim/sulfamethoxazole.

Agar disk diffusion testing was carried out on the isolates following standard protocols and the interpretive guidelines from CLSI to determine the susceptibility profiles of the isolates. ${ }^{19}$ The following antimicrobial agents were tested: amoxicillin/clavulanic acid (20/10 $\mu \mathrm{g} /$ disk), ampicillin (10 $\mu \mathrm{g} /$ disk), amikacin (30 $\mu \mathrm{g} / \mathrm{disk})$, ceftiofur (30 $\mu \mathrm{g} / \mathrm{disk})$, ceftriaxone $(30 \mu \mathrm{g} / \mathrm{disk})$, cefoxitin $(30 \mu \mathrm{g} / \mathrm{disk})$, ciprofloxacin (5 $\mu \mathrm{g} /$ disk), chloramphenicol (30 $\mu \mathrm{g} /$ disk), gentamicin (10 $\mu \mathrm{g} /$ disk $)$, kanamycin (30 $\mu \mathrm{g} /$ disk $)$, nalidixic acid (30 $\mu \mathrm{g} /$ disk $)$, streptomycin $(10 \mu \mathrm{g} / \mathrm{disk})$, sulfamethoxazole (300 $\mu \mathrm{g} / \mathrm{disk})$, tetracycline $(30 \mu \mathrm{g} / \mathrm{disk})$, and trimethoprim/sulfamethoxazole (1.25/23.75 $\mu \mathrm{g} / \mathrm{disk})$. Following 18 to 20 hours of incubation at $35^{\circ} \mathrm{C}$, the plates were examined and the zone of inhibition measured for each antibiotic.

The manual broth microdilution susceptibility testing was performed using susceptibility plates prepared in the test laboratory. Two-fold serial dilutions of the antimicrobial agents were added to the wells of a 96-well microtiter plate. The following agents were tested (dilution ranges): amoxicillin/clavulanic acid $(2 / 1 \mu \mathrm{g} / \mathrm{ml}$ to $64 / 32 \mu \mathrm{g} / \mathrm{ml})$, 
ampicillin (0.5-1024 $\mu \mathrm{g} / \mathrm{ml})$, amikacin $(4-128 \mu \mathrm{g} / \mathrm{ml})$, apramycin $(0.5-1024 \mu \mathrm{g} / \mathrm{ml})$, ceftriaxone $(2-64 \mu \mathrm{g} / \mathrm{ml})$, cefoxitin $(2-64 \mu \mathrm{g} / \mathrm{ml})$, cephalothin $(2-64 \mu \mathrm{g} / \mathrm{ml})$, ciprofloxacin $(0.5-1024 \mu \mathrm{g} / \mathrm{ml})$, chloramphenicol $(0.5-1024$ $\mu \mathrm{g} / \mathrm{ml})$, gentamicin $(0.5-1024 \mu \mathrm{g} / \mathrm{ml})$, kanamycin, (0.5-1024 $\mu \mathrm{g} / \mathrm{ml})$ nalidixic acid $(4-128 \mu \mathrm{g} / \mathrm{ml})$, streptomycin $(0.5-1024$ $\mu \mathrm{g} / \mathrm{ml})$, sulfamethoxazole $(4-128 \mu \mathrm{g} / \mathrm{ml})$, tetracycline $(0.5-1024$ $\mu \mathrm{g} / \mathrm{ml})$ and trimethoprim/sulfamethoxazole $(0.24 / 4.8 \mu \mathrm{g} / \mathrm{ml}$ to $7.68 / 153.6 \mu \mathrm{g} / \mathrm{ml})$. Bacterial suspensions were prepared by diluting cells in sterile Mueller-Hinton broth and the cell density was adjusted to $5 \times 10^{5} \mathrm{CFU} / \mathrm{ml}$. The cell suspensions were added to the plates (total volume/well $=50 \mu \mathrm{l}$ ). The plates were incubated for 18 to 20 hours at $35^{\circ} \mathrm{C}$ and the growth (turbidity) was measured at $600 \mathrm{~nm}$.

The Sensititre automated antimicrobial susceptibility system (Trek Diagnostic Systems, Westlake, $\mathrm{OH}$ ) was also used for broth microdilution testing and interpreted according to CLSI guidelines for broth microdilution methods. ${ }^{21}$ Sensititre susceptibility testing was performed according to the manufacturer's instructions with the CMV1AGNF plates (Trek Diagnostics) utilized by NARMS. Each plate contained the following drugs: amoxicillin/clavulanic acid, ampicillin, amikacin, ceftiofur, ceftriaxone, cefoxitin, ciprofloxacin, chloramphenicol, gentamicin, kanamycin, nalidixic acid, streptomycin, sulfamethoxazole, tetracycline and trimethoprim/sulfamethoxazole.

Vitek testing was carried out using commercially available veterinary drug cards (GNS-207) according to the manufacturer's instructions (BioMérieux, Inc., Durham, NC). The drugs included in the panel were amoxicillin/clavulanic acid, ampicillin, amikacin, carbenicillin, ceftiofur, cephalothin, ciprofloxacin, chloramphenicol, enrofloxacin, gentamicin, nitrofurantoin, piperacillin, tetracycline, ticarcillin, tobramycin and trimethoprim/sulfamethoxazole. Interpretive criteria (extrapolated from CLSI guidelines) provided by the manufacturer were used to interpret the results.

\section{Quality Control Testing}

For the agar disk diffusion testing, Escherichia coli (ATCC 25922) was used as the quality control strain for testing. For the broth microdilution and Vitek methods, E. coli (ATCC 25922), Staphylococcus aureus (ATCC 29213), Enterococcus faecalis (ATCC 29212) and Pseudomonas aeruginosa (ATCC 27853) were used as quality control strains. Quality control testing was performed for each method on the day AST testing was performed and the results were interpreted as described by CLSI. If the test results for the quality control strains were outside the prescribed control ranges, all susceptibility testing performed on that day was repeated. The AST methods and the agar disk diffusion testing were performed in laboratories affiliated with the study's principal investigators (Nayak and Foley) and the results compared to ensure agreement of test methodology for the drugs being studied.

\section{Data Analysis}

Data analysis for the experiments was carried out as described by Guthrie et al, ${ }^{13}$ with some modifications. The AST results for the isolates studied were interpreted according to the guidelines set by CLSI for agar disk diffusion, manual broth microdilution, and Sensititre microdilution. The Vitek results used were those reported by the Vitek system as susceptible, intermediate-susceptible, or resistant. The agar disk diffusion method was utilized as a reference method in this study. The interpreted results were compared to determine the percent agreement among the test method and the reference, and to determine any discrepancies among the results. The discrepancies were classified as being very major errors if the isolate was interpreted as susceptible by the method being evaluated and resistant by the reference method. A major error was detected if the isolate was interpreted as resistant by the method being evaluated and susceptible by the reference method, and the discrepancy was considered a minor error if an isolate was classified as intermediate-susceptible by either the test or reference method and the other test classified the isolate as either resistant or susceptible. ${ }^{13}$

\section{Results}

For each result reported in the study, the quality control organisms were calculated to be within the acceptable ranges for the different antimicrobial agents tested. When the two investigators compared the agar disk diffusion results to one another, there were four minor discrepancies, which were resolved upon a repeated disk diffusion test. Each discrepancy involved an isolate being classified as intermediate resistance to gentamicin by one laboratory and resistant by the other. The cumulative study results are reported in table 1 . All isolates in the study were identified as susceptible to amikacin or ciprofloxacin by each method; therefore, the results for these antimicrobials were not included in table 1 . Resistance to amoxicillin/clavulanic acid $(n=3)$, ampicillin $(n=2)$, and chloramphenicol $(n=3)$ was only detected by the Sensititre method. Resistance to gentamicin $(\mathrm{n}=24)$, tetracycline $(\mathrm{n}=10)$ and trimethoprim/sulfamethoxazole $(\mathrm{n}=5)$ was detected by multiple methods. Overall, the AST result agreement among all four methods for the drugs tested was amikacin (100\%), amoxicillin/clavulanic acid (96.1\%), ampicillin (97.1\%), chloramphenicol (96.2\%), ciprofloxacin $(100 \%)$, gentamicin $(80.0 \%)$, tetracycline $(80.0 \%)$ and trimethoprim/sulfamethoxazole (94.3\%). A number of instances of non-agreement for amoxicillin/clavulanic acid $(\mathrm{n}=6 ; 5.7 \%)$, gentamicin $(n=7 ; 6.7 \%)$ and tetracycline $(n=12 ; 11.4 \%)$ are due to isolates calculated to be intermediately susceptible.

In a comparison of the overall error rates among the different methods and antimicrobial agents, there were a limited number of very major errors for most of the agents tested. The exceptions were with the Vitek, in which six $(5.7 \%)$ very major errors for gentamicin and five $(4.8 \%)$ for tetracycline were detected. There were no major errors detected for the Vitek; however, there were major errors in the results obtained by Sensititre and manual broth microdilutions. The major error rate for Sensititre was highest for gentamicin 
Table 1. Comparison of susceptibility testing method results to agar diffusion reference.

\begin{tabular}{|c|c|c|c|c|c|c|}
\hline \multirow[b]{2}{*}{ Methods } & \multirow[b]{2}{*}{ Classification } & \multirow[b]{2}{*}{ No. (\%) } & \multirow[b]{2}{*}{$\begin{array}{l}\text { Agreement to } \\
\text { reference: number (\%) }\end{array}$} & \multicolumn{3}{|c|}{$\begin{array}{c}\text { Categorical Discrepancies from } \\
\text { Reference: Number (\%) }\end{array}$} \\
\hline & & & & $\begin{array}{l}\text { Very major } \\
\text { error }\end{array}$ & $\begin{array}{l}\text { Major } \\
\text { error }\end{array}$ & $\begin{array}{l}\text { Minor } \\
\text { error }\end{array}$ \\
\hline \multicolumn{7}{|c|}{ Amoxicillin/clavulanic acid } \\
\hline \multirow[t]{4}{*}{ Agar diffusion* } & $S$ & $105(100)$ & & & & \\
\hline & I & $0(0)$ & & & & \\
\hline & $\mathrm{R}$ & $0(0)$ & & & & \\
\hline & Total & $105(100)$ & & & & \\
\hline \multirow[t]{4}{*}{ Manual dilution } & $\mathrm{S}$ & $105(100)$ & $105(100)$ & & & \\
\hline & I & $0(0)$ & N/A & & & \\
\hline & $\mathrm{R}$ & $0(0)$ & $\mathrm{N} / \mathrm{A}$ & & & \\
\hline & Total & $105(100)$ & $105(100)$ & 0 & 0 & 0 \\
\hline \multirow{4}{*}{ Sensititre } & $\mathrm{S}$ & $96(91.1)$ & $96(91.1)$ & & & \\
\hline & I & $6(5.7)$ & $0(0)$ & & & \\
\hline & $\mathrm{R}$ & $3(2.9)$ & $0(0)$ & & & \\
\hline & Total & $105(100)$ & $96(91.1)$ & 0 & $3(2.9)$ & 0 \\
\hline \multirow[t]{4}{*}{ Vitek } & $\mathrm{S}$ & $105(100)$ & $105(100)$ & & & \\
\hline & I & $0(0)$ & N/A & & & \\
\hline & $\mathrm{R}$ & $0(0)$ & $\mathrm{N} / \mathrm{A}$ & & & \\
\hline & Total & $105(100)$ & $105(100)$ & 0 & 0 & 0 \\
\hline \multicolumn{7}{|l|}{ Ampicillin } \\
\hline \multirow[t]{4}{*}{ Agar diffusion* } & $S$ & $105(100)$ & & & & \\
\hline & I & $0(0)$ & & & & \\
\hline & $\mathrm{R}$ & $0(0)$ & & & & \\
\hline & Total & $105(100)$ & & & & \\
\hline \multirow[t]{4}{*}{ Manual dilution } & $\mathrm{S}$ & 105 (100) & $105(100)$ & & & \\
\hline & I & $0(0)$ & N/A & & & \\
\hline & $\mathrm{R}$ & $0(0)$ & $\mathrm{N} / \mathrm{A}$ & & & \\
\hline & Total & $105(100)$ & $105(100)$ & & & \\
\hline \multirow[t]{4}{*}{ Sensititre } & $\mathrm{S}$ & $103(97.1)$ & $103(97.1)$ & 0 & 0 & 0 \\
\hline & I & $1(1.0)$ & $0(0)$ & & & \\
\hline & $\mathrm{R}$ & $2(1.9)$ & $0(0)$ & & & \\
\hline & Total & $105(100)$ & $103(97.1)$ & & & \\
\hline \multirow[t]{5}{*}{ Vitek } & $S$ & 105 (100) & $105(100)$ & 0 & $2(1.9)$ & 0 \\
\hline & 1 & $0(0)$ & N/A & & & \\
\hline & $\mathrm{R}$ & $0(0)$ & $\mathrm{N} / \mathrm{A}$ & & & \\
\hline & Total & $105(100)$ & $105(100)$ & & & \\
\hline & & & & 0 & 0 & 0 \\
\hline \multicolumn{7}{|l|}{ Chloramphenicol } \\
\hline \multirow[t]{4}{*}{ Agar diffusion* } & $\mathrm{S}$ & $104(99.0)$ & & & & \\
\hline & I & $0(0)$ & & & & \\
\hline & $\mathrm{R}$ & $1(1.0)$ & & & & \\
\hline & Total & $105(100)$ & & & & \\
\hline \multirow[t]{4}{*}{ Manual dilution } & $\mathrm{S}$ & 105 (100) & $104(99.0)$ & & & \\
\hline & I & $0(0)$ & N/A & & & \\
\hline & $\mathrm{R}$ & $0(0)$ & $\mathrm{N} / \mathrm{A}$ & & & \\
\hline & Total & $105(100)$ & $104(99.0)$ & 0 & 0 & 0 \\
\hline
\end{tabular}

Table 1 continues on next page. 
Table 1 (continued). Comparison of susceptibility testing method results to agar diffusion reference.

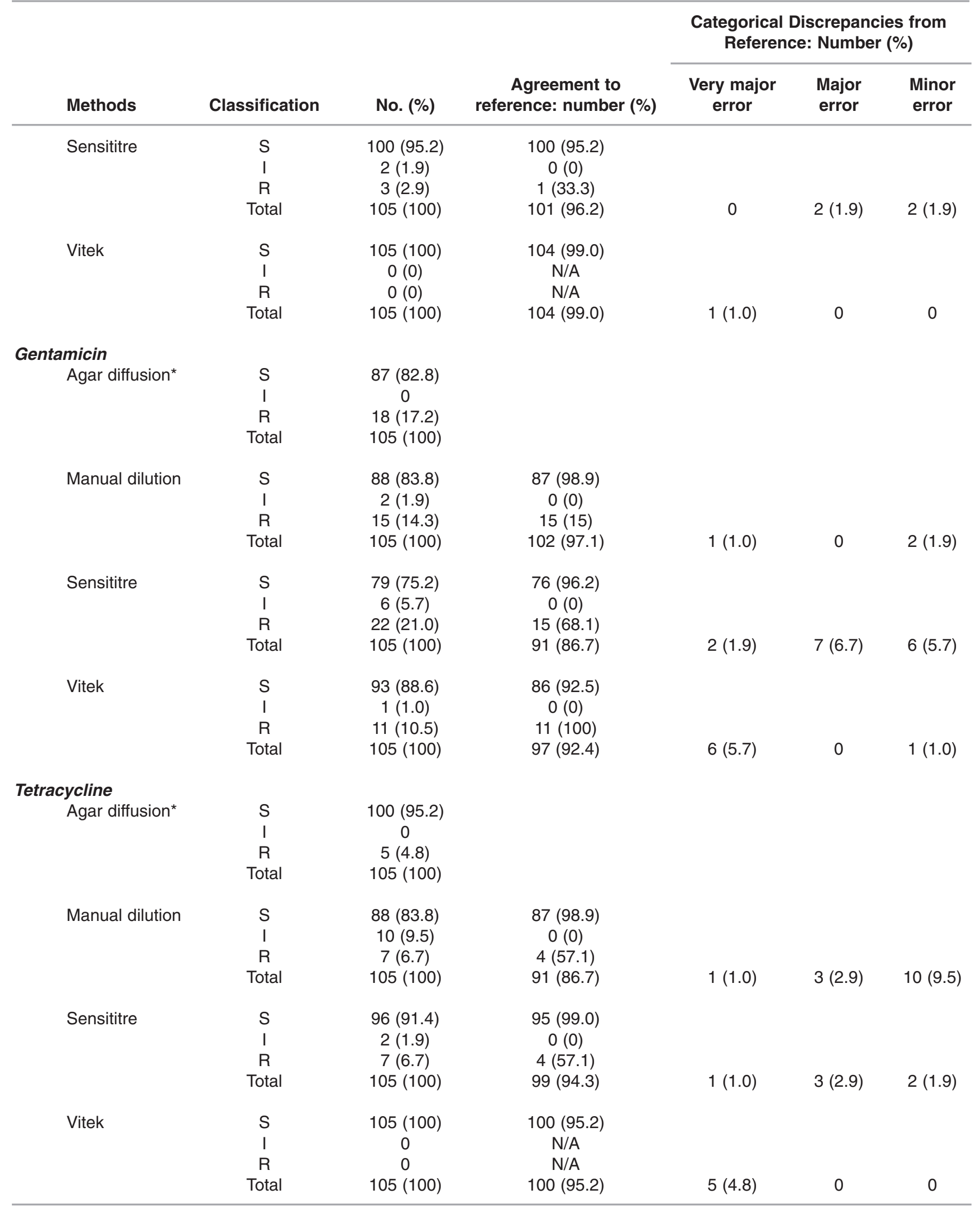

Table 1 continues on next page. 
Table 1 (continued). Comparison of susceptibility testing method results to agar diffusion reference.

\begin{tabular}{|c|c|c|c|c|c|c|}
\hline \multirow[b]{2}{*}{ Methods } & \multirow[b]{2}{*}{ Classification } & \multirow[b]{2}{*}{ No. (\%) } & \multirow[b]{2}{*}{$\begin{array}{l}\text { Agreement to } \\
\text { reference: number (\%) }\end{array}$} & \multicolumn{3}{|c|}{$\begin{array}{c}\text { Categorical Discrepancies from } \\
\text { Reference: Number (\%) }\end{array}$} \\
\hline & & & & $\begin{array}{l}\text { Very major } \\
\text { error }\end{array}$ & $\begin{array}{l}\text { Major } \\
\text { error }\end{array}$ & $\begin{array}{l}\text { Minor } \\
\text { error }\end{array}$ \\
\hline \multicolumn{7}{|c|}{ Trimethoprim/sulfamethoxazole } \\
\hline \multirow[t]{4}{*}{ Agar diffusion* } & $\mathrm{S}$ & $103(98.1)$ & & & & \\
\hline & 1 & $1(1.0)$ & & & & \\
\hline & $\mathrm{R}$ & $1(1.0)$ & & & & \\
\hline & Total & $105(100)$ & & & & \\
\hline \multirow[t]{4}{*}{ Manual dilution } & $\mathrm{S}$ & 98 (93.3) & $98(100)$ & & & \\
\hline & 1 & $2(1.9)$ & 0 & & & \\
\hline & $\mathrm{R}$ & $5(4.8)$ & $1(20)$ & & & \\
\hline & Total & $105(100)$ & $99(94.3)$ & 0 & $3(2.9)$ & $3(2.9)$ \\
\hline \multirow[t]{4}{*}{ Sensititre } & $S$ & $102(97.1)$ & $102(100)$ & & & \\
\hline & I & 0 & & & & \\
\hline & $\mathrm{R}$ & $3(2.9)$ & 1 (33.3) & & & \\
\hline & Total & $105(100)$ & $103(98.1)$ & 0 & $1(1.0)$ & $1(1.0)$ \\
\hline \multirow[t]{4}{*}{ Vitek } & $\mathrm{S}$ & 105 (100) & $102(97.1)$ & & & \\
\hline & 1 & 0 & $\mathrm{~N} / \mathrm{A}$ & & & \\
\hline & $\mathrm{R}$ & 0 & $\mathrm{~N} / \mathrm{A}$ & & & \\
\hline & Total & $105(100)$ & $102(97.1)$ & $1(1.0)$ & 0 & $1(1.0)$ \\
\hline
\end{tabular}

* Reference method utilized in the study. S, sensitive; I, intermediate; R, resistant.

$(n=7 ; 6.7 \%)$ and tetracycline $(n=3 ; 2.9 \%)$, and in the manual broth microdilutions, the major error rate was the highest for tetracycline $(n=3 ; 2.9 \%)$. The highest minor error rate was detected in the manual broth microdilution with tetracycline $(n=10 ; 9.5 \%)$ and in Sensititre with gentamicin $(n=6 ; 5.7 \%)$. Overall, there was $97.1 \%, 95.5 \%$ and $98.0 \%$ agreement with the reference disk diffusion method for manual broth microdilution, Sensititre microdilution and the Vitek methods, respectively.

\section{Discussion}

In this study, we compared the interpretation of results from four different AST methods for their ability to detect resistance in $S$. enterica serovar Heidelberg. In general, the results indicated that the AST methods correlated with one another, with a few exceptions. The most significant discrepancies among the methods generally fell into two categories; the first was the detection of a lower number of resistance phenotypes with the Vitek system, which led to an elevated very major error rate. Overall, the number of very major errors in the study was $18(2.1 \%)$ among the 840 drug/strain combinations studied, 13 (72.2\%) of which occurred with the Vitek system. The second general category of discrepancy was a higher number of resistance phenotypes detected with the Sensititre method, which led to an elevated major error rate. There were $24(2.9 \%)$ major errors, 18 $(75.0 \%)$ of which occurred with the Sensititre system. There were also a number of minor errors detected in the study that were more widely distributed among the various typing methods. Of the 28 (3.3\%) minor errors, 15 (53.6\%) occurred with the manual broth microdilution, 11 (39.3\%) with the Sensititre microdilution, and $2(7.1 \%)$ with the Vitek system. These discrepancies, in part, may be due to the interpretation of the results, because in a number of cases the resistance detected was just over the MIC resistance breakpoint, and the susceptible isolates were detected just below the intermediate-susceptible range with other methods. While there were some discrepancies, overall there was a greater than 95\% agreement between each testing method and the reference method.

When the results of this study were compared to other AST comparison studies, the results were relatively similar. The error rates reported by Guthrie et al ${ }^{13}$ had a similar pattern to the present study. In the majority of drugs tested with Streptococcus pneumoniae, there were few errors. However, there was an elevation in the number of very major and major errors for trimethoprim/sulfamethoxazole. ${ }^{13}$ The pattern of these findings were similar to what was seen for gentamicin and tetracycline in the present study, which had higher error rates compared to the rest of the drugs tested. Studies that examined susceptibility testing in gram negative organisms also had similar results to the current study, with overall categorical error rates of around $2 \%$ for Vitek and broth microdilution testing, which was similar to the $2.1 \%$ to $3.3 \%$ range in our study. ${ }^{22,23}$ When a consortium in Europe 
compared disk diffusion, broth microdilution and agar breakpoint testing with Salmonella isolates, they found a high level of agreement among the results of each method to detect resistant organisms. ${ }^{24}$ These findings were similar to our results for the most part, with the exception of gentamicin and tetracycline, which had higher error rates in our study. The finding that gentamicin and tetracycline had the highest error rates in our study was not unique. Other multi-method AST study findings for gram negative organisms have found similar findings for gentamicin and tetracycline compared to the other drugs tested. ${ }^{25,26}$

The finding of only a single very major and a single major error for trimethoprim/sulfamethoxazole was notable because trimethoprim/sulfamethoxazole is one of the agents of choice for the treatment of invasive salmonellosis. ${ }^{27}$ Results indicating that gentamicin and tetracycline had the highest error rates were also interesting because these drugs are not typically used for the treatment of salmonellosis, thus the elevated error levels in the testing of these agents will likely have limited impact on clinical outcomes. Taken together, if the findings of ideal drugs have the lowest and highest error rates for Salmonella, they would likely be similar to the results of our study, with the more clinically important drugs (such as trimethoprim/sulfamethoxazole and ciprofloxacin) having low error rates and those used less frequently, if at all (gentamicin and tetracycline), having the higher error rates. The results of this study demonstrated that even though the methods have been thoroughly validated by CLSI and method developers, there could be some variability among laboratory personnel. Overall, the study confirmed that the interpreted results of the methods were similar for susceptibility testing of $S$. enterica serovar Heidelberg isolates with some noted exceptions. Therefore, under most circumstances, the interpreted results of the susceptibility testing methods evaluated in this study can be compared to results of other testing methods, potentially permitting greater sharing of susceptibility testing results among scientists and diagnosticians.

\section{References}

1. Mead PS, Slutsker L, Dietz V, McCaig LF, Bresee JS, Shapiro C, Griffin PM, Tauxe RV. Food-related illness and death in the United States. Emerg Infect Dis 1999;5:607-625.

2. CDC. Salmonella Surveillance: Annual Summary, 2003. Atlanta, GA: US Department of Health and Human Services, CDC, 2004. Available at: http://www.cdc.gov/ncidod/dbmd/phlisdata/salmtab/2003/Salm onellaAnnualSummary2003.pdf. Accessed February 27, 2007.

3. Centers for Disease Control and Prevention (CDC). Preliminary FoodNet data on the incidence of infection with pathogens transmitted commonly through food-10 States, United States, 2005. MMWR Morb Mortal Wkly Rep 2006;55:392-395.

4. Ferris KE, Aalsburg AM, Iseminger GR. Salmonella serotypes from animals and related sources during July 2001-June 2002. Proc U S Animal Health Assoc 2002;106:467-497.
5. Zhao S, McDermott PF, Friedman S, Abbott J, Ayers S, Glenn A, Hall-Robinson E, Hubert SK, Harbottle H, Walker RD, Chiller TM, White DG. Antimicrobial resistance and genetic relatedness among Salmonella from retail foods of animal origin: NARMS retail meat surveillance. Foodborne Pathog Dis 2006;3:106-117.

6. Logue CM, Sherwood JS, Olah PA, Elijah LM, Dockter MR. The incidence of antimicrobial-resistant Salmonella spp on freshly processed poultry from US Midwestern processing plants. J Appl Microbiol 2003;94:16-24.

7. Nayak R, Stewart T, Wang RF, Lin J, Cerniglia CE, Kenney PB. Genetic diversity and virulence gene determinants of antibiotic-resistant Salmonella isolated from preharvest turkey production sources. Int J Food Microbiol 2004;91:51-62.

8. Food Availability: Custom Queries. Economic Research Service/United States Department of Agriculture Web site. Available at: http://www.ers.usda.gov/Data/FoodConsumption/FoodAvailQ ueriable.aspx. Accessed November 11, 2006.

9. Zhao S, White DG, Friedman SF, Glenn A, Ayers SL, Abbott JW, Hubert SK, Hall-Robinson E, Harbottle HC, Walker RD, McDermott PF. Characterization of Salmonella Heidelberg from retail meat samples: National Antimicrobial Resistance Monitoring System (NARMS): 2002-2004. Proceedings from the 10th Annual PulseNet Update Meeting, Miami, FL. 2006.

10. Aarestrup FM, Hasman H, Olsen I, Sorensen G. International spread of bla (CMY-2)-mediated cephalosporin resistance in a multiresistant Salmonella enterica serovar Heidelberg isolate stemming from the importation of a boar by Denmark from Canada. Antimicrob Agents Chemother 2004;48:1916-1917.

11. Marano NN, Rossiter S, Stamey K, Joyce K, Barrett TJ, Tollefson LK, Angulo FJ. The National Antimicrobial Resistance Monitoring System (NARMS) for enteric bacteria, 1996-1999: surveillance for action. J Am Vet Med Assoc 2000;217:1829-1830.

12. Clinical and Laboratory Standards Institute (CLSI) (formerly National Committee for Clinical Laboratory Standards (NCCLS)). Performance Standards for Antimicrobial Susceptibility Testing: Sixteenth Informational Supplement M100-S16 (ISBN 1-56238-588-7). Wayne, PA: CLSI; 2006.

13. Guthrie LL, Banks S, Setiawan W, Waites KB. Comparison of MicroScan MICroSTREP, PASCO, and Sensititre MIC panels for determining antimicrobial susceptibilities of Streptococcus pneumoniae. Diagn Microbiol Infect Dis 1999;33:267-273.

14. O'Hara CM, Tenover FC, Miller JM. Parallel comparison of accuracy of API 20E, Vitek GNI, MicroScan Walk/Away Rapid ID, and Becton Dickinson Cobas Micro ID-E/NF for identification of members of the family Enterobacteriaceae and common gram-negative, non-glucose-fermenting bacilli. J Clin Microbiol 1993;31:3165-3169.

15. Steward CD, Mohammed JM, Swenson JM, Stocker SA, Williams PP, Gaynes RP, McGowan JE Jr, Tenover FC. Antimicrobial susceptibility testing of carbapenems: multicenter validity testing and accuracy levels of five antimicrobial test methods for detecting resistance in Enterobacteriaceae and Pseudomonas aeruginosa isolates. J Clin Microbiol 2003;41:351-358.

16. Varkey P, Poland GA, Cockerill FR 3rd, Smith TF, Hagen PT. Confronting bioterrorism: physicians on the front line. Mayo Clin Proc 2002;77:661-672.

17. Critchley IA, Karlowsky JA. Optimal use of antibiotic resistance surveillance systems. Clin Microbiol Infect 2004; 10:502-511.

18. Enserink M. Bioterrorism. Researchers question obsession with Cipro. Science 2001;294:759-761. 
19. National Committee for Clinical Laboratory Standards (NCCLS). Performance Standards for Antimicrobial Disk and Dilution Susceptibility Tests for Bacteria Isolated from Animals; Approved Standard-Second Edition. NCCLS document M31-A2 (ISBN 1-56238-461-9). Wayne, PA: NCCLS; 2002.

20. Torok TJ, Tauxe RV, Wise RP, Livengood JR, Sokolow R, Mauvais S, Birkness KA, Skeels MR, Horan JM, Foster LR. A large community outbreak of salmonellosis caused by intentional contamination of restaurant salad bars. JAMA 1997;278:389-395.

21. National Committee for Clinical Laboratory Standards (NCCLS). Performance Standards for Antimicrobial Susceptibility Testing; Twelfth Informational Supplement. NCCLS document M100-S12 (ISBN 1-56238-454-6). Wayne, PA: NCCLS; 2002.

22. Doern GV, Brueggemann AB, Perla R, Daly J, Halkias D, Jones RN, Saubolle MA. Multicenter laboratory evaluation of the bioMerieux Vitek antimicrobial susceptibility testing system with 11 antimicrobial agents versus members of the family Enterobacteriaceae and Pseudomonas aeruginosa. J Clin Microbiol 1997;35:2115-2119.

23. Karlowsky JA, Weaver MK, Thornsberry C, Dowzicky MJ, Jones ME, Sahm DF. Comparison of four antimicrobial susceptibility testing methods to determine the in vitro activities of piperacillin and piperacillin-tazobactam against clinical isolates of Enterobacteriaceae and Pseudomonas aeruginosa. J Clin Microbiol 2003;41:3339-3343.

24. Threlfall EJ, Fisher IS, Ward LR, Tschape H, Gerner-Smidt P. Harmonization of antibiotic susceptibility testing for Salmonella: results of a study by 18 national reference laboratories within the European Union-funded Enter-net group. Microb Drug Resist 1999;5:195-200.

25. Staneck JL, Glenn S, DiPersio JR, Leist PA. Wide variability in Pseudomonas aeruginosa aminoglycoside results among seven susceptibility testing procedures. J Clin Microbiol 1989;27:2277-2285.

26. Reiber NE, Kelly MT, Latimer JM, Tison DL, Hysmith RM. Comparison of the Cathra Repliscan II, the AutoMicrobic system Gram-Negative General Susceptibility-Plus Card, and the Micro-Media System Fox Panel for dilution susceptibility testing of gram-negative bacilli. J Clin Microbiol 1985;21:959-962.

27. Gilbert DN, Moellering RC, Sande, MA. The sanford guide to antimicrobial therapy, 33rd ed. Hyde Park, VT: Antimicrobial Therapy; 2003.

\section{Author Affiliations}

Rajesh Nayak, PhD

National Center for Toxicological Research

United States Food and Drug Administration

Jefferson, Arkansas

Veronica Call, BS

Office of Regulatory Affairs/Arkansas Regional Laboratory

United States Food and Drug Administration

Jefferson, Arkansas

Pravin Kaldhone, $M S$

National Farm Medicine Center

Marshfield Clinic Research Foundation

Marshfield, Wisconsin and

Department of Biology

University of Central Arkansas

Conway, Arkansas
Cynthia Tyler, MS

National Farm Medicine Center

Marshfield Clinic Research Foundation

Marshfield, Wisconsin and

Department of Biology

University of Central Arkansas

Conway, Arkansas

Gwendolyn Anderson, BS

Office of Regulatory Affairs/Arkansas Regional Laboratory United States Food and Drug Administration

Jefferson, Arkansas

Sarah Phillips, PhD

Office of Regulatory Affairs/Arkansas Regional Laboratory

United States Food and Drug Administration

Jefferson, Arkansas

Khalil Kerdahi, BS

Office of Regulatory Affairs/Arkansas Regional Laboratory

United States Food and Drug Administration

Jefferson, Arkansas

Steven L. Foley, PhD

Office of Regulatory Affairs/Arkansas Regional Laboratory

United States Food and Drug Administration

Jefferson, Arkansas and

National Farm Medicine Center

Marshfield Clinic Research Foundation

Marshfield, Wisconsin and

Department of Biology

University of Central Arkansas

Conway, Arkansas 